\title{
Advanced musculoskeletal simulation as an ergonomic design method
}

\author{
John Rasmussen ${ }^{\mathrm{a}}$, Mark Boocock ${ }^{\mathrm{b}, \mathrm{c}}$ and Gunther Paul ${ }^{\mathrm{b}}$ \\ ${ }^{a}$ M-Tech, Aalborg University, Fibigerstraede 16, DK-9220 Aalborg East, Denmark. \\ ${ }^{\mathrm{b}}$ Ergolab, Mawson Institute, University of South Australia, University Boulevard, Mawson Lakes SA 5095, \\ Australia \\ ${ }^{c}$ Auckland University of Technology, Auckland, New Zealand
}

\begin{abstract}
This paper investigates new opportunities for ergonomic design based on advanced computer simulated musculoskeletal modeling. The approach is inspired by the influence that other advanced computer-aided engineering technologies have had on product design. The background to the musculoskeletal modeling approach (AnyBody Modeling System) is briefly explained and illustrated by means of a hand sawing task. Simple design modifications to the handle of the saw provide empirical data about muscle forces on which designers can use to enhance product design. It is concluded that musculoskeletal simulation has the potential to significantly improve ergonomic design of many tools and products that derive their value from interfacing with the human body.
\end{abstract}

Keywords: Musculoskeletal simulation, Ergonomic design, Anybody Modeling System, Hand saw. Design

\section{Introduction}

The ability to simulate physical phenomena to a high level of detail and accuracy using computers has revolutionized the world. Very few of the products used in modern day life could have been designed and produced to their current performance and quality in the absence of advanced computer simulation.

Computer-Aided Engineering (CAE) tools, such as finite element methods, finite volume methods and boundary element methods, are essentially for solving differential equations which allow the designer to evaluate a range of physical properties, e.g. solid mechanics, fluid mechanics, thermal and electromagnetic properties. Advancements in this technology have now progressed to the extent that it allows most types of technical products and production processes to be addressed.

The success of many products is often governed by peoples' ability to interact or access an item. Such products affect all aspects of daily life, including, for instance, hand tools, machinery and furniture at work, land and water vehicles, leisure and sports equipment, medical and assistive devices, apparel and humanpowered vehicles like wheelchairs. Simulating human interaction with these products is complicated by the fact that the human body is a complex, dynamic system, controlled not merely by a prescribed sequence of actions, but to a large extent by voluntary decisions and complex reactions of the central nervous system. Therefore, ergonomic studies have not been influenced by CAE technology to the same extent as engineering design.

In recent years, the advent of new tools [1-3] for musculoskeletal simulation has increased the potential for significantly improving the ergonomic design process and ergonomic assessment of design. In this paper we investigate the use of one such tool, 'The AnyBody Modeling System', applied to solve a oneparameter and yet, complex ergonomic design problem. The aim of this paper is to investigate the potential of computer-aided musculoskeletal modeling in the ergonomic design process, in the same way as CAE technology has had for engineering design.

\section{Methods}

A musculoskeletal model of a human operator using a hand saw was constructed in the AnyBody Modeling System (ver. 4.1, AnyBody Technology A/S, Denmark). The AnyBody Modeling System 
uses recorded motion as input into the model. Inverse dynamics and a muscle recruitment algorithm, based on optimization, are then used to predict redundant muscle actions, and/or joint moments responsible for the motion. This entails solving a series of dynamic equilibrium equations within the biomechanical model which leads to the calculation of joint reaction forces, mechanical work and other properties that are useful to the assessment of human performance. Please refer to Damsgaard et al. [1] for a more detailed explanation of the analytical approach.

Muscle systems are considered redundant in the sense that they contain more muscles than the required degrees-of-freedom (DoF) and, as such, are over-actuated. This means that there are an infinite number of different muscle patterns that can balance the external load, and the central nervous system (CNS) selects one according to the situation. Musculoskeletal modeling systems are faced with the challenge of mimicking the choices made by the CNS and selecting physiologically realistic muscle activation patterns appropriate to each time step in the given task. For the sake of numerical feasibility, the algorithm must be efficient.

In terms of a muscle modeling, a minimum muscle fatigue criterion which seeks to minimize the maximum relative load on a muscle can be supported by physiological and evolutionary arguments. Such fatigue criteria have the added benefit that they can be placed into a mathematical form that allows them to be solved by a series of linear programming problems [4]. This not only provides numerical efficiency, but has the attractive feature of muscles sharing the same levels of activation which can be combined into an 'envelope' of all muscle activations. From a physiological point-of-view, this means that fatigue of the first muscle will be delayed, as far as possible, and that most muscles will tire simultaneously. The resulting envelope of muscle activation becomes an excellent criterion for determining the effort involved in performing a particular movement or task, and offers an opportunity to compare different scenarios when designing tools and workplaces, as will be demonstrated below.

\section{Case study}

\subsection{Hand saw design}

The design of hand saws has changed significantly over the years, due to the advent of new materials and production methods. Traditional wood working saws, often referred to as frame saws or bow saws, have flexible blades suspended in a wooden frame. These saws are often treasured by many traditional woodworkers for their ergonomic qualities.

However, most modern carpenters use panel saws or fish saws with a simpler design. They comprise a tapered blade that is rigid enough so that it does not require them to be suspended in a frame and can be driven by a simple handle attached to one end. Modern production methods mean that these saws can be manufactured with plastic handles, and in large quantities at a low cost. Furthermore, unlike bow saws they have unlimited cutting depth and can reach locations that cannot be accessed with a bow saw. Fig. 1 illustrates the two types of saws.



Figure 1: A classical bow saw and a modern fish saw.

In terms of their design, the two saws differ significantly with regard to the location of the handle and posture that the user adopts. This has a significant impact on physiological effort and the ease-ofuse. Fish saws have the handle placed at the end of the blade, such that the blade becomes an elongation of the forearm. In contrast, bow saws provide a bar supported parallel above the blade which the user grasps. These two types of grip create fundamentally different biomechanical properties during the sawing motion. To demonstrate how advanced computer musculoskeletal modeling can nowadays be used to assist in the design process, a simulation of a sawing task involving the fish saw was performed using the AnyBody musculoskeletal modeling system. This simulation involved a simple sawing task in which the effects on muscular effort arising from small changes in the placement of the handle were investigated. 


\subsection{Task simulation and muscle modeling}

The simulated task of sawing using a fish saw involved one forward stroke of the saw over a distance of $20 \mathrm{~cm}$. This motion was performed at constant velocity for 1 second. A constant force was considered to act with $5 \mathrm{~N}$ tangential and $3.33 \mathrm{~N}$ normal to the line of the blade. The relationship between normal and tangential forces will in practice depend on the design of the blade (e.g. shape and size of the teeth) and its sharpness, and the angle of the blade in relation to the cutting plane. The model was constructed such that forces were always directed tangentially (perpendicularly to the blade), even though the blade is known to slightly change direction over the course of the motion due to changes in hand/arm orientation. It is also important to point out that the design optimization problem used in the musculoskeletal modeling approach involved constant mechanical work and therefore, constant sawing, irrespective of changes to other parameters within the model.

The work piece (e.g. wooden block) on which the blade contacted was a fixed object in space located in front of the body, and the saw was constrained to slide over its surface. The right hand of the operator was connected to the handle of the saw and the left hand was holding onto the environment to assist with upper body support. The model comprised of the upper body and pelvis, which was rotated 30 degrees about a vertical axis, causing the body to be posi-

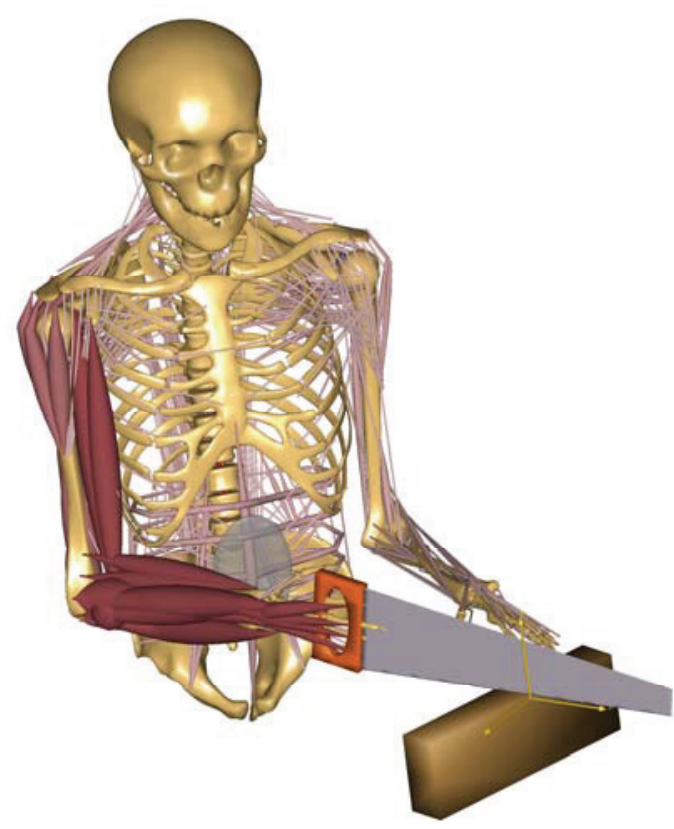

Figure 2: The musculoskeletal model. tioned slightly side-on to the work piece, as is commonly observed when operating hand saws using self-selected postures. Recommendations regarding best practice indicate that the saw should "run in a straight line with the forearm so that it becomes a continuation of the arm. It is important, too, that the user stand in such position that his elbow and shoulder are in direct line with the saw" [9].

The model was restricted to a single design parameter, i.e. the handle's offset perpendicularly to the blade. As mentioned earlier, fish saws are usually designed such that the blade becomes an extension of the user's forearm. Using the offset parameter, the position of the handle could be varied such that forearm was either below, on or above the height of the blade. Fig. 2 shows the model in the basic configuration, i.e. with the handle placed centrally and aligned with the end of the blade (corresponding to zero vertical displacement).

The muscle configuration of the trunk and upper extremities is based on models previously reported in the literature [6-8], and comprises approximately 400 individually activated muscles. The connection point for applying power from the palm of the hand to the handle of the saw was at the center of the hand, adjacent to the last three fingers.

\subsection{Analysis}

Data are presented for the muscle activation envelopes. As explained earlier, this envelope expresses the level of muscle activation anywhere in the body and is a representation of combined muscle activity. Therefore, it serves as a meaningful physiological measure of the risk of developing fatigue in the trunk and upper extremities when performing repetitive movement of the saw, as well as a measure of perceived effort on the part of the user. With all things being equal, a lower muscle activity envelope would be representative of lower levels of perceived effort on behalf of the user. Hence, an optimal saw design is one that is considered to result in a minimum muscle activation envelope for the same amount of physical work.

Analyses were performed with variable handle offsets of -50, 0, 50,100,150, 200, 250 and $300 \mathrm{~mm}$, where positive values designate handle positions above the blade. Fig. 3 shows the relative muscle activity envelopes for each of these handle positions.

It is evident from Figure 3 that it becomes easier for the operator to advance the saw as the blade moves forward over the work piece. It can also be seen that the muscles are overloaded (i.e. above $100 \%$ relative muscle activity) at the beginning of the stroke for all handle positions. Under normal 
circumstances, the operator is likely to manage this situation by reducing the normal force acting on the saw in order to advance the saw.

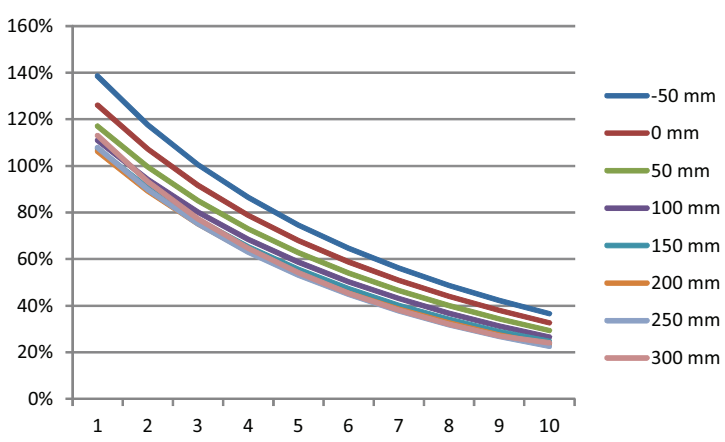

Figure 3: Muscle activity envelopes for different handle offsets, representing relative muscle activity [\%] as a function of time [-].

The analysis also reveals that the effort required to drive the movement forward depends on the height of the handle above or below the blade, and that an increase or reduction in relative muscle activity with handle displacement occurs across the entire movement, i.e. none of the curves cross over each other. This means that the effort of driving the saw can be represented by the effort at a single point in the movement. This effect can be clearly seen by plotting the muscle activity envelope at the beginning of the stroke against the offset position of the handle (Fig. 4). It appears that an optimum offset exists at a handle position of approximately $200 \mathrm{~mm}$, and also that this optimum is rather flat in the sense that handle offsets between $150 \mathrm{~mm}$ and $250 \mathrm{~mm}$ result in almost similar levels of muscle activation. This is positive from a design point-of-view, in that it allows designers to select an optimum handle position based on other factors relating to the design. In addition, this finding supports production tolerance.

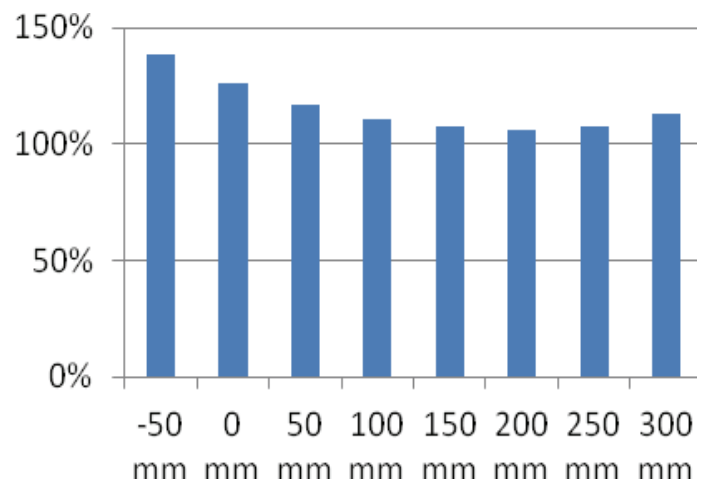

Figure 4: Relative muscle activity [\%] envelope at the start of the stroke as a function of each handle offset.

\section{Discussion}

The musculoskeletal modeling example of the sawing task suggests that it is advantageous from an ergonomic perspective to move the handle position of the saw upwards with respect to the blade, such that the blade is not directly extending the forearm, but located somewhat below it. A normal fish saw has the handle placed at the end of the blade and does not allow for such a hand position. The grip proposed by the musculoskeletal modeling approach is consistent with the normal grip used with the classical bow saw described earlier and shown in Fig. 1.

The saw example investigated here was chosen due to its simple design which lends itself to illustrate the benefits of CAE based on musculoskeletal models. Despite the single parameter of handle location used in this example, the underlying mechanics is much more complex and much less understood by traditional ergonomic approaches, such as physiological and psychophysical experimentation. Methods involving human subjects are expensive and time-consuming to perform. In experiment based studies similar to this, a significant number of subjects would be involved, requiring a number of saws with different handle designs.

In addition to muscle effort, the musculoskeletal model described here also computes joint forces, individual muscle forces and metabolism, overall metabolic efficiency, joint angle variation and many other relevant reactions of the human body to the external load. Thus, the same methods can be used to investigate other types of ergonomic problems, notably issues related to work place design and risks of load-induced injuries.

Musculoskeletal simulation appears to offer the potential to substantially improve the field of ergonomic design and assessment in the same way that, for instance, finite element analysis has fundamentally changed the engineering design process.

\section{References}

[1] M. Damsgaard, J. Rasmussen, S.T. Christensen, E. Surma, and M.d. Zee, Analysis of Musculoskeletal Systems in the AnyBody Modeling System, Simulation Modelling Practice and Theory, vol 14, 2006, pp. 1100-1111.

2] A. Veloso, G. Esteves, S. Silva, C. Ferreira, F. Brandão, Biomechanics modeling of human musculoskeleial system using ADAMS multibody dynamics package, Proceedings of the Fourth IASTED International Conference on Biomedical Engineering, Int. Assoc. of Science and Technology for Development, 2006, pp. 401-407. 
[3] S.L. Delp, F.C. Anderson, A.S. Arnold, P. Loan, A. Habib, C.T. John, E. Guendelman and D.G. Thelen, OpenSim: Open-source software to create and analyze dynamic simulations of movement, IEEE Transactions on Biomedical Engineering, Vol. 54, pp. 1940-1959, November 2007.

[4] J. Rasmussen, M. Damsgaard and M. Voigt, Muscle recruitment by the $\min / \max$ criterion: a comparative numerical study, Journal of Biomechanics, Vol. 34, pp. 409-415.

[5] J. Rasmussen, M. Damsgaard, S.T. Christensen and E. Surma, Design optimization with respect to ergonomic properties, Structural and Multidisciplinary Optimization, Vol. 24, 2002, pp. 87-97.

[6] L. Hansen, M.d. Zee, J. Rasmussen, T.B. Andersen, C. Wong and C E.B. Simonsen, Anatomy and biome- chanics of the back muscles in the lumbar spine with reference to biomechanical modeling, Spine Vol. 31, 2006, pp. 1888-1899.

[7] M. d. Zee, L. Hansen, C. Wong, J. Rasmussen and E.B Simonsen, A generic detailed rigid-body lumbar spine model, Journal of Biomechanics, Vol. 40, 2007, pp. 1219-1227.

[8] F.C. Van der Helm, H.E. Veeger, G.M. Pronk, L.H. Van der Woude and R.H. Rozendal, Geometry parameters for musculoskeletal modelling of the shoulder system, Journal of Biomechanics Vol. 25, 1992, pp. $129-144$.

[9] Disston Tool Manual for School Shops, Henry Disston \& Sons, 1927. 